that others will take up the investigation as its solution seems to depend upon a multiplicity of observation.

If we find in our cases of recent glaucoma, cultures of staphylococci from the uvea, which differ consistently from those obtained from the conjunctiva, we cannot, a priori, exclude the staphylococcus as a potent factor in the disease.

Montreal.

Yours truly,

RICHARD KERRY.

\title{
THE LARGE LENS OF THE GULLSTRAND SLIT-LAMP
}

\section{To the Editor of The British Journal of Ophthalmology}

SIR,-Those familiar with the slit-lamp will be aware that only an elongated vertically disposed central portion of the lens referred to above is made use of, the portions of the lens on either side of this being superfluous. The area used is defined by the aperture in the diaphragm, which elsewhere covers the superfluous portions of the lens. The lens is of large diameter $(50 \mathrm{~mm}$.) in order that it may receive rays, emerging from the slit-diaphragm, covering a relatively wide vertical angle. The lens being accurately corrected, and being of such large diameter, produces a good intensity of focal illumination. Even so, the intensity with the ordinary Nernst-filament slit-lamp leaves no spare power to waste; for high power work, the intensity with this lamp sets many limitations.

The large cross-diameter of the lens is undesirable, as most owners of the apparatus must have experienced, because it prevents the illuminator being brought to a sufficiently acute angle with the objectives of the microscope to enable the observer thus to view the deeper parts of the vitreous, only the vitreous just behind the lens and not that which is deeper being directly accessible to view. The present method of looking deeper into the vitreous is to make an elaborate readjustment of the instrument and employ Koeppe's reflecting mirror to procure the deeper view. This mirror is a very necessary accessory for certain procedures, and familiarity with its use is called for; but when time is limited it is a disadvantage to be compelled to make a gross readjustment of the apparatus every time that a rather deeper glance into the vitreous is desired.

There are, moreover, other instances, in routine observation of more anterior parts of the eye, in which facility for a simple change to an acute angle between the axes of illumination and observation is desirable. This is rendered feasible by Dr. Vogt's "small 
lens," the diameter of which is much smaller than that of the large Gullstrand lens; but the objection I have experienced in the use of this small lens is that the intensity of the focal illumination is diminished in proportion with the reduction in the diameter of the lens, and even with the Gullstrand lens there is, as I have suggested above, already but little light to spare.

1 have, therefore, had a lens made which in shape and dimensions resembles the large Gullstrand lens with its superfluous lateral portions ground away; this lens serves the purposes served by the Gullstrand lens, and in addition permits of adjustment between the axis of observation and illumination to a much more acute angle. The small Vogt lens is not highly corrected for aberration because such correction is unnecessary for the smaller vertical angle of the fewer incident rays which it collects; but efficient correction is required in the larger Gullstrand lens, and it is this that makes it expensive.

Readers may care to know that Messrs. C. Davis Keeler have kindly offered to grind down the Gullstrand lens to the shape I have indicated at no more than what they suggest is but a very small risk of damage to the lens in the process of grinding. One precaution I would add-the transverse width of the product should somewhat exceed the measurement set by the present diaphragm of the Gullstrand lens, to avoid the possibility of any rays from the lateral portion of the transmitted beam impinging at the grazing angle on the flattened sides, and so being internally reflected at the critical angle, with disturbing results ; and for that matter, the sides need not be made quite flat. The lens is mounted in a holder of suitable width.

LoNDON,

Yours truly, Basit. Graves.

August 14, 1923.

\section{NOTES}

WE regret to announce the death of KARL $\mathrm{v}$. Deaths Hess, of Munich. Details of the life work of this distinguished man will be published in

a later number.

Dr. M. R. LöwEGReN, who founded the first eye clinic in Sweden, has died at the age of 87 years.

Mr. C. L. Gimblett has been appointed Appointment ophthalmic surgeon to the Royal Northern Hospital, London. 